\title{
Desarrollo de una PCR múltiple para la identificación de Staphylococcus spp. como causa de mastitis caprina
}

\author{
Development of a multiplex PCR for the identification of Staphylococcus spp. \\ as a cause of caprine mastitis
}

RA Ruiz-Romero ${ }^{\mathrm{a} *}$, RA Cervantes-Olivares ${ }^{\mathrm{b}}$, D Martínez-Gómez, ${ }^{\mathrm{c}}$ AE Ducoing-Watty, ${ }^{\mathrm{d}}$ L Hernández-Andrade ${ }^{\mathrm{e}}$

\author{
aLaboratorio de Microbiología, Departamento de Medicina y Zootecnia de Rumiantes, \\ Facultad de Medicina Veterinaria y Zootecnia, Universidad Nacional Autónoma de México, México, D.F. \\ 'Laboratorio de Micología, Departamento de Microbiología e Inmunología, \\ Facultad de Medicina Veterinaria y Zootecnia, Universidad Nacional Autónoma de México, México, D.F. \\ 'Laboratorio de Microbiología Agropecuaria, División de Ciencias Biológicas y de la Salud \\ de la Universidad Autónoma Metropolitana-Xochimilco, México, D.F. \\ ${ }^{\mathrm{d} D}$ Departamento de Medicina y Zootecnia de Rumiantes, Facultad de Medicina Veterinaria y Zootecnia, \\ Universidad Nacional Autónoma de México, México, D.F. \\ eLaboratorio de Bacteriología, Centro Nacional de Investigación Disciplinaria en Microbiología Animal \\ del Instituto Nacional de Investigaciones Forestales y Agropecuarias, México, D.F.
}

\begin{abstract}
SUMMARY
"Mastitis" refers to inflammation of the mammary gland. Despite the economic losses that it causes in dairy goats, there is scarce information about the epidemiological status of the disease in Mexico. The aim of this study was to develop a multiplex polymerase chain reaction (PCR) test to diagnose Staphylococcus agents involved in caprine mastitis. Milk samples from animals in intensive and semi-intensive production systems were taken from two farms located at Tequisquiapan, Querétaro, Mexico. The samples were obtained from healthy goats. The multiplex PCR was developed to identify coagulase-negative Staphylococcus and S. aureus. To achieve the identification of Staphylococcus spp, primers for the 16s rRNA region were used. Another two pair of primers were used for $S$. aureus, the first pair corresponded to the clfA gene and the second pair corresponded to the $S$. aureus coa gene. Multiplex PCR was performed in 30 milk samples and it was possible to detect S. aureus and coagulase-negative Staphylococcus. Further studies are needed to evaluate the sensitivity and specificity of this technique.
\end{abstract}

Key words: mastitis, coagulase-negative Staphylococci, Staphylococcus aureus, multiplex PCR.

\section{RESUMEN}

La mastitis se refiere a la inflamación de la glándula mamaria, a pesar de las pérdidas económicas que produce en cabras lecheras, existe poca información acerca de la situación epidemiológica de la enfermedad en México. El objetivo de este estudio fue desarrollar una reacción en cadena de la polimerasa (PCR) múltiple para el diagnóstico de Staphylococcus spp como agente involucrado en mastitis caprina. Se colectaron muestras de leche de cabras clínicamente sanas en sistemas de producción intensivo y semi-intensivo de dos granjas ubicadas en Tequisquiapan, Querétaro, México. La PCR múltiple se desarrolló para identificar a los Staphylococcus coagulasa-negativos (SCN) y a Staphylococcus aureus. Para lograr la identificación del género Staphylococcus spp se utilizó el par de iniciadores correspondientes a la región 16s RNAr, para el caso de S. aureus se utilizaron 2 pares de iniciadores, el primer par de iniciadores corresponden al gen clfA y el segundo par corresponden al gen coa de S. aureus. Se realizó la PCR múltiple a 30 muestras de leche de cabra en donde se pudo detectar a S. aureus y a SCN, sin embargo, estos estudios son preliminares por lo que se requiere de estudios posteriores para evaluar la sensibilidad y especificidad de esta técnica.

Palabras clave: mastitis, Staphylococcus coagulasa-negativos, Staphylococcus aureus, PCR múltiple.

\section{INTRODUCCIÓN}

La primera consecuencia de la mastitis es una disminución en la producción de leche así como cambios patológi-

Aceptado: 28.03.2013.

\# Financiado por el Proyecto PAPIIT (Programa de Apoyo a Proyectos de Investigación e Innovación Tecnológica) IN218708.

*rarr2212@unam.mx cos en la ubre (Shearer y col 2003). En estudios realizados en México, los microorganismos aislados con mayor frecuencia en 565 cabras lecheras ordeñadas mecánicamente localizadas en Guanajuato, fueron Staphylococcus aureus (32,6\%), Staphylococcus epidermidis $(32,6 \%)$ y Micrococcus $\operatorname{spp}(30,4 \%)$, el 4,4\% restante correspondió a Streptococcus uberis, Streptococcus dysgalactiae y Escherichia coli (Amezcua 1981). En el año 1998 en un estudio realizado en Puebla con 113 cabras con mastitis subclínica se de- 
terminó que las frecuencias asociadas a esta condición eran: Staphylococcus cohnii, Staphylococcus capiti, S. aureus, y en menor cantidad Alcaligenes faecalis, Mannheimia haemolytica, Acinetobacter spp, Bacillus spp y Corynebacterium spp (Bonilla y col 1998). En el 2008, se analizaron 118 muestras de leche de cabras criadas en la ciudad de México y ordeñadas de forma mecánica, donde el 64,5\% (76 muestras) resultaron positivas a mastitis subclínica, de estas 76 muestras el 81,5\% resultaron negativas y el 18,5\% resultaron positivas donde se aisló únicamente a Staphylococcus spp. (Figueroa y col 2008). En el año 2010, en el municipio de Tequisquiapan, Querétaro, México, se trabajaron 484 muestras de leche de cabras en donde el 18,60\% (90 muestras) fueron positivas a mastitis subclínica, los géneros bacterianos que se aislaron fueron los Staphylococcus coagulasa negativos (SCN) (63.92\%), donde las 3 principales especies aisladas fueron Staphylococcus chromogenes $(48,39 \%)$, Staphylococcus simulans $(22,58 \%)$ y Staphylococcus xylosus (20.97\%), el resto de los aislados correspondieron a Staphylococcus sciuri, Staphylococcus haemolyti-

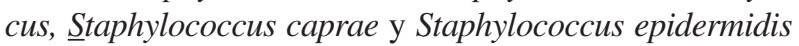
$(8,06 \%)$ el segundo género y especie que se aisló con mayor frecuencia fue Streptococcus uberis $(10,31 \%)$ y por último Staphylococcus aureus (5,15\%). (Ruiz 2010). El género Staphylococcus se puede dividir en Staphylococcus coagulasa positivos (CPS) y Staphylococcus coagulasa negativos de acuerdo a su capacidad para coagular el plasma sanguíneo (Hermans y col 2004, Pyörälä y col 2009, Watanabe y col 2009). En la mayoría de los laboratorios clínicos, los métodos de identificación se basan en pruebas bioquímicas de bacterias aisladas de leche (Phuektes y col 2001, Gillespie y col 2005), sin embargo, el análisis microbiológico de la leche no garantiza el aislamiento de bacterias debido a que los animales con mastitis subclínica pueden eliminar al microorganismo causante de manera intermitente durante la lactación, así como el uso de antibióticos, presencia de leucocitos y un alto contenido de células somáticas (SC) en la leche que inhiben el crecimiento bacteriano in vitro (Clavijo y col 2002). Debido a estas limitaciones, se han desarrollado técnicas como la reacción en cadena de la polimerasa (PCR) múltiple que consigue amplificar simultáneamente y en un único tubo diferentes secuencias blanco, permitiendo la detección e identificación simultánea de distintos genes de interés (Markoulatos y col 2002, Méndez y col 2004). En este estudio se desarrolló y estandarizó una PCR múltiple para ofrecer una alternativa más precisa y rápida para el diagnóstico de Staphylococcus spp implicados en esta enfermedad.

\section{MATERIAL Y MÉTODOS}

\section{CARACTERÍSTICAS DE LAS MUESTRAS}

Se colectaron treinta muestras de leche de cabras provenientes de dos granjas ubicados en Tequisquiapan, Querétaro, México, las cabras estaban clínicamente sanas al momento de muestreo en diferentes etapas de la lactancia y con una condición corporal media, el ordeño se realizaba de forma mecánica dos veces al día. La toma de muestras consistió en la desinfección de los pezones con torunda y alcohol al $70 \%$ y la extracción manual y desecho de los tres primeros chorros de leche de cada una de las glándulas, posteriormente, se obtuvieron $30 \mathrm{ml}$ de leche de las dos glándulas en frascos nuevos, estériles y herméticos, fueron trasladadas a temperatura de refrigeración al Laboratorio de Microbiología Agropecuaria en el Departamento de Producción Agrícola y Animal de la Universidad Autónoma Metropolitana-Xochimilco, Ciudad de México, México para su análisis bacteriológico.

\section{ANÁLISIS BACTERIOLÓGICO}

Las muestras se agitaron en vórtex para homogenizarla y liberar las bacterias que pudieran encontrarse atrapadas en los glóbulos de grasa. Se realizó un frotis directo y se llevó a cabo la tinción de Gram. Con el fin de identificar únicamente bacterias mesofílicas, aerobias facultativas, se colocaron $30 \mu \mathrm{l}$ de la muestra en una placa de agar sangre y agar MacConkey en la primera estría para realizar el primocultivo, ambas placas se incubaron a $37^{\circ} \mathrm{C}$ durante $24 \mathrm{~h}$ en condiciones de aerobiosis, en caso de no observar crecimiento se incubaron durante 48 h más hasta descartar crecimiento bacteriano. Los cultivos fueron examinados para determinar la morfología macroscópica de las colonias desarrolladas y su número relativo de acuerdo al número de cuadrantes del agar en donde se observó crecimiento bacteriano. A partir de las colonias representativas se realizó un frotis fijo teñido con Gram para guiar la identificación bioquímica recomendada (Carter y col 1984). En cuanto a los cocos Gram positivos catalasa positivos se realizaron las pruebas bioquímicas correspondientes para identificarlos como Staphylococcus spp o Micrococcus spp; en el caso de pertenecer al género Staphylococcus se realizó la prueba de coagulasa en tubo para identificarlos como Staphylococcus coagulasa positivo ( $S$. aureus o $S$. intermedius) o Staphylococcus coagulasa negativos. En el caso de los cocos Gram positivos catalasa negativos se identificaron como Streptococcus y con la prueba de Christie, Atkins y Munch-Petersen (CAMP) - esculina se llegó a la especie ( $S$. agalactiae, S. dysgalactiae o $S$. uberis), los bacilos Gram positivos con presencia de espora fueron identificados como Bacillus spp y por último los bacilos Gram negativos oxidasa negativo fueron identificados como enterobacterias llegando al género con las pruebas bioquímicas recomendadas (Carter y col 1984).

\section{EXTRACCIÓN DE ADN}

Para dar validez a la prueba de la PCR múltiple se utilizaron cepas de referencia del American Type Culture Collection (ATCC) como Staphylococcus aureus ATCC 
29737 y Staphylococcus xylosus ATCC 700404. Para obtener ADN de las cepas bacterianas y de las muestras de leche de cabra se realizó el procedimiento descrito previamente (Cremonesi y col 2006) con modificaciones; en el caso de las cepas bacterianas, se inocularon 5 colonias en $15 \mathrm{ml}$ de caldo infusión cerebro corazón (CICC) para cada cepa y se incubaron a $37{ }^{\circ} \mathrm{C}$ en agitación durante $18 \mathrm{~h}$, transcurrido este tiempo cada muestra de $15 \mathrm{ml}$ se centrifugó durante $10 \mathrm{~min}$ a $600 \mathrm{~g}$, descartando el sobrenadante, en el caso de las muestras de leche, se centrifugaron $10 \mathrm{ml}$ durante $10 \mathrm{~min}$ a $600 \mathrm{~g}$ eliminando el sobrenadante, a partir de este paso, las pastilla obtenida de cada muestra se resuspendió en $500 \mu \mathrm{l}$ de solución salina estéril $(\mathrm{NaCl})$ al $0,9 \%$ y se centrifugó nuevamente durante $15 \mathrm{~min}$ a $450 \mathrm{~g} \mathrm{a} 4^{\circ} \mathrm{C}$ eliminando el sobrenadante, este paso se realizó una vez más. La pastilla se suspendió en $50 \mu \mathrm{l}$ de solución salina y se agregaron $300 \mu \mathrm{l}$ de solución de lisis ( $3 \mathrm{M}$ isotiocianato de guanidina, $20 \mathrm{mM} \mathrm{EDTA} \mathrm{pH}$ $8,10 \mathrm{mM}$ Tris- $\mathrm{HCl} \mathrm{pH}$ 6,8, tritón $40 \mathrm{mg} / \mathrm{ml}$, ditiotreitol $10 \mathrm{mg} / \mathrm{ml}$ ) y $200 \mu \mathrm{l}$ de solución de unión $(40 \mathrm{mg} / \mathrm{ml}$ de silica resuspendida en solución de lisis), se homogenizaron $15 \mathrm{~s}$ en vórtex y se dejaron reposar 5 min a temperatura ambiente. Transcurrido este tiempo se centrifugó a $30 \mathrm{~s} \mathrm{a}$ $450 \mathrm{~g}$ eliminando el sobrenadante, se realizaron 2 lavados añadiendo $200 \mu \mathrm{l}$ de solución de lisis homogenizando perfectamente y se centrifugó $30 \mathrm{~s}$ a $450 \mathrm{~g}$ eliminando el sobrenadante, se realizaron 2 lavados más con $200 \mu \mathrm{l}$ de solución de lavado (25\% etanol absoluto, $25 \%$ isopropanol, $100 \mathrm{mM} \mathrm{NaCl}, 10 \mathrm{mM}$ Tris-HCl pH 8) centrifugando con las mismas constantes anteriores, se realizó un último lavado con $200 \mu \mathrm{l}$ de etanol absoluto centrifugando nuevamente, se eliminó el sobrenadante y se secó la pastilla durante $10 \mathrm{~min}$ a $37^{\circ} \mathrm{C}$, se añadieron $50 \mu \mathrm{l}$ de solución de elusión (10 mM Tris-HCl pH 8, 1 mM EDTA pH 8) y se incubaron en baño María durante $15 \min$ a $65^{\circ} \mathrm{C}$. Por último se centrifugó 5 min a $600 \mathrm{~g}$ y se transfirió el sobrenadante a un tubo limpio.

\section{PCR MÚLTIPLE}

Para lograr la identificación del género Staphylococcus se utilizaron los iniciadores correspondientes a la región 16s del ácido ribonucleico ribosomal (ARNr) la cual es única para este género en comparación con otras eubacterias (Mason y col 2001) Para el caso de S. aureus se utilizaron 2 pares de iniciadores, el primer par corresponden al gen clfA exclusivo de $S$. aureus y el segundo par corresponden al gen coa de $S$. aureus el cual codifica para la enzima coagulasa, la secuencia de cada par de iniciadores se indican más adelante (cuadro 1). Cada reacción de la PCR múltiple, consistió en $45 \mu \mathrm{l}$ de PCR Supermix ${ }^{1}$, suplementado con $10 \mu \mathrm{l}$ de Taq polimerasa ${ }^{2}$ ( $5 \mathrm{U} / \mu \mathrm{l}$ ) por $500 \mu \mathrm{L}$ de PCR Supermix, $2 \mu \mathrm{l}$ de los ini-

Invitrogen No. Catálogo 10572014, Carlsbad, California 92008, USA.

2 Invitrogen No. Catálogo 10342053, Carlsbad, California 92008, USA. ciadores del gen coa a una concentración de $20 \mu \mathrm{M}, 0.5$ $\mu \mathrm{l}$ de los iniciadores del gen clfA y de la región $16 \mathrm{~s}$ de Staphylococcus spp a una concentración de $5 \mu \mathrm{M}$ y $1 \mu \mathrm{l}$ de ADN de $S$. aureus a una concentración de $100 \mathrm{ng} / \mu \mathrm{l}$; además, con cada reacción, se utilizaron 2 controles positivos: en el primero se utilizaron $2 \mu \mathrm{l}$ de ADN de $S$. $a u$ reus ATCC 29737 a una concentración de 10 ng/ $\mu \mathrm{l}$ y en el segundo control positivo se utilizaron $2 \mu \mathrm{l}$ de ADN de S. xylosus ATCC 700404 a la misma concentración que el anterior y como blanco se sustituyó el ADN por $1 \mu \mathrm{l}$ de agua inyectable estéril, en cada uno de los controles se utilizaron las cantidades estandarizadas de los iniciadores. El protocolo del termociclador ${ }^{3}$ consistió en un ciclo inicial de desnaturalización a $95{ }^{\circ} \mathrm{C}$ por $5 \mathrm{~min}, 30$ ciclos de desnaturalización a $95^{\circ} \mathrm{C}$ por $1 \mathrm{~min}$, alineamiento $56{ }^{\circ} \mathrm{C}$ por $1 \min 30 \mathrm{~s}$, extensión a $72{ }^{\circ} \mathrm{C}$ por 2 min y finalmente un ciclo de extensión final a $72{ }^{\circ} \mathrm{C}$ por $5 \mathrm{~min}$.

\section{RESULTADOS Y DISCUSIÓN}

Se logró estandarizar la PCR múltiple para el género Staphylococcus spp utilizando los iniciadores de la región 16 RNAr y de los genes clfA y coa con ADN extraído de leche de cabra positiva a $S$. aureus y/o a SNC por bacteriología tradicional, como se muestra en el gel de agarosa al 1,5\% (figura 1). El diagnóstico se realizó en 30 muestras de leche de cabra de las cuales 11 fueron positivas a todas las bandas $(36,67 \%), 3$ muestras fueron positivas a la región 16s RNAr y al gen clfA (10\%), una muestra fue positiva únicamente a la banda del gen clfA $(3,33 \%)$ y 15 muestras fueron negativas $(50 \%)$ (cuadro 2). Fue posible desarrollar y estandarizar la técnica de PCR múltiple para $S$. aureus y SCN realizando el diagnóstico de estos agentes en aproximadamente 6 horas. Para asegurar la identificación del género Staphylococcus spp, se utilizaron los iniciadores para la región 16s RNAr que corresponde a la subunidad menor ribosomal. Estos genes se conservan entre los Staphylococcus spp y son únicos en comparación con otras eubacterias (Mosbath y col 2005). Para la identificación específica de $S$. aureus fueron utilizados iniciadores para el gen clfA y el gen coa, aunque otras especies de Staphylococcus son capaces de producir coagulasa libre o unida, estos iniciadores son específicos para la secuencia del gen de S. aureus (Mason y col 2001). Se pudieron integrar los 3 pares de iniciadores alcanzando una temperatura óptima de alineación a $56{ }^{\circ} \mathrm{C}$. La PCR múltiple tiene la ventaja de detectar más de una secuencia blanco ahorrando tiempo y dinero en el diagnóstico; a pesar de utilizarse con éxito en el diagnóstico de varias enfermedades; también existen varias desventajas como presentar una sensibilidad y especificidad mucho más baja que una PCR simple, esto puede deberse a la competencia que existen por los desoxiribonucleóti-

Termociclador Corbett Research Modelo CG1-96. 27220 Turnberry Lane Suite 200 Valencia, CA 91355, USA. 
Cuadro 1. Secuencias de los iniciadores para cada gen.

Primer sequence for each target gene.

\begin{tabular}{ccc}
\hline Gen & Forward $\left(5^{\prime}-3^{\prime}\right)$ & Reverse $\left(5^{\prime}-3^{\prime}\right)$ \\
\hline $\begin{array}{c}\text { 16s rRNA } \\
\text { Staphlococcus spp } \\
\text { clfA }\end{array}$ & CCTATAAGACTGGGATAACTTCGGG & CTTTGAGTTTCAACCTTGCGGTCG \\
S. aureus & GCAAAATCCAGCACAACAGGAAACGA & CTTGATCTCCAGCCATAATTGGTGG \\
co s aureus & CCTGTACCAGCATCTCTATATTTAA & CAAAGCAGATGCGATAGTAAC \\
\hline
\end{tabular}

Cuadro 2. Resultados de bacteriología.

Bacteriology results.

\begin{tabular}{|c|c|c|c|}
\hline $\begin{array}{l}\text { Número de } \\
\text { muestra }\end{array}$ & Bacteriología & $\begin{array}{l}\text { Bandas } \\
\text { esperadas }\end{array}$ & $\begin{array}{c}\text { Bandas } \\
\text { obtenidas }\end{array}$ \\
\hline 1 & S. aureus & 3 & 3 \\
\hline 2 & S. aureus & 3 & 3 \\
\hline 3 & S. aureus & 3 & 3 \\
\hline 4 & S. simulans & 1 & 3 \\
\hline 5 & S. simulans & 1 & 3 \\
\hline 6 & S. simulans & 1 & 3 \\
\hline 7 & S. simulans & 1 & 3 \\
\hline 8 & $\begin{array}{l}\text { S.simulans, } \\
\text { E. aerogenes }\end{array}$ & 1 & 3 \\
\hline 9 & S. chromogenes & 1 & 3 \\
\hline 10 & $\begin{array}{l}\text { S. simulans, } \\
\text { S. xylosus }\end{array}$ & 1 & 3 \\
\hline 11 & E. aerogenes & 0 & 3 \\
\hline 12 & $\begin{array}{c}\text { Bacillus, } \\
\text { E. aerogenes, } \\
\text { S. simulans }\end{array}$ & 1 & 2 \\
\hline 13 & Negativo & 0 & 2 \\
\hline 14 & Bacillus & 0 & 2 \\
\hline 15 & S. chromogenes & 1 & 1 \\
\hline 16 & S. uberis & 0 & 0 \\
\hline 17 & S. xylosus & 1 & 0 \\
\hline 18 & S. simulans & 1 & 0 \\
\hline 19 & E. aerogenes & 0 & 0 \\
\hline $20-30$ & Negativo & 0 & 0 \\
\hline
\end{tabular}

dos trifosfato y la taq polimerasa cuando varios pares de iniciadores se combinan en una sola reacción (Phuektes y col 2001), posiblemente por esto, se obtuvo un solo caso en el que solo amplificó la banda correspondiente a $c l f a$. Existieron 3 casos en los que el gen coa no se detectó a pesar de la amplificación de la región 16s del RNAr y del gen clfA, sin embargo, a pesar de que se ha informado de la existencia de cepas coagulasa negativos de $S$. $a u$ -

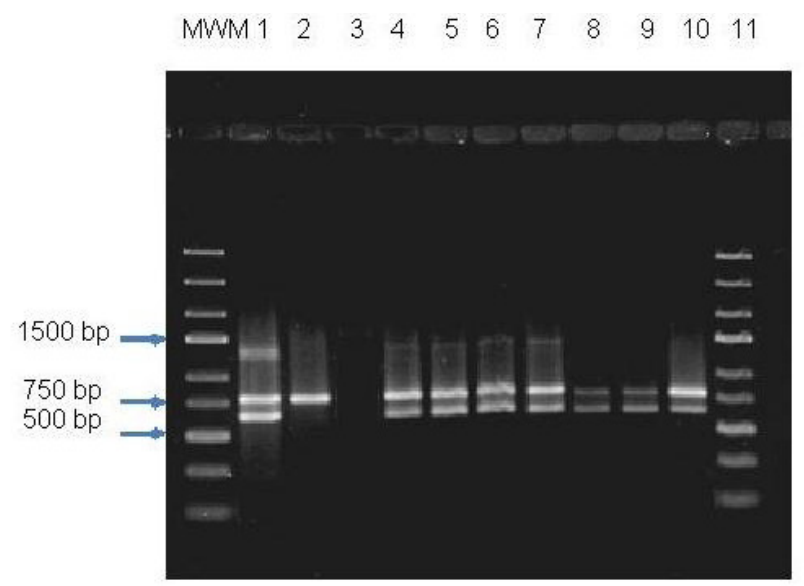

Figura 1. Fotografía de un gel de agarosa al $1.5 \%$ teñido con bromuro de etidio donde se muestra PCR Múltiplex para $S$. aureus y SCN. MPM = marcador de peso molecular Gene Ruler Express, 1 = Control positivo con ADN de $S$. aureus ATCC 29737, 2 = Control positivo con ADN de $S$. xylosus ATCC 700404, 3 = Control negativo, 4-7 = PCR multiplex con ADN de leche mamitosa de cabra, 8-10 = Muestras positivas a la región 16 s y al gen clfA.

Photograph of a $1.5 \%$ agarose gel stained with ethidium bromide showing multiplex PCR for $S$. aureus and CNS, MWM = Gene Ruler Express DNA ladder, 1 = positive control with DNA from $S$. $a u$ reus ATCC 29737, 2 = positive control with DNA from S. xylosus ATCC 700404, 3 = negative control, 4-7 = PCR multiplex from some samples using DNA extracted from goat milk, $8-10=$ positive samples to $16 \mathrm{~s}$ and clfA gene.

reus, debido a mutaciones en el gen que se asocia con la pérdida de la capacidad de producir coagulasa (Korman 1963, Taponen y col 2009), hay pocos estudios sobre el porcentaje de aislados que carecen del gen coa, algunos autores mencionan que únicamente el $1 \%$ de las cepas de S. aureus carecen de este gen (Barret 1983), solamente en el caso de haberse logrado el aislamiento bacteriológico de estas cepas se podría realizar la prueba de coagulasa en tubo, por lo que los iniciadores utilizados para este gen pueden estar inhibiéndose y se necesitan más pruebas para determinar la concentración indicada para realizar esta PCR múltiple, a pesar de que la banda 16s ARNr amplifica utilizando como testigo el ADN de $S$. xylo- 
sus ATCC 700404, no hubo ningún caso en el que sólo se detectaran a los SCN, lo que puede indicar probable existencia en la misma muestra de ADN de $S$. aureus y $\mathrm{SCN}$, por lo que se amplificó la misma banda de la región 16s, además de la detección de los genes específicos para S. aureus. A pesar de que este PCR múltiple detectó patógenos bacterianos que no fueron detectados en el aislamiento bacteriológico, es necesario seguir probando esta técnica con más muestras de leche de cabra para determinar la sensibilidad y especificidad de esta prueba.

\section{REFERENCIAS}

Amezcua MA. 1981. Prevalencia de mastitis subclínica en hatos caprinos en la zona central del Bajío. Tesis licenciatura, Facultad de Medicina Veterinaria y Zootecnia, Universidad Nacional Autónoma de México, Distrito Federal, México.

Barret S. 1983. Rate of isolation of Staphylococcus aureus strains possessing coagulase or clumping factor. Eur J Microbiol Infect Dis 2, 475-476.

Bonilla CS, M Rosas, A Hernández, A Díaz, G Villa, Z Hernández. 1998. Agentes etiológicos involucrados en la mastitis subclínica en cabras lecheras. Memorias del XXVII Congreso Nacional de Buiatría, Facultad de Medicina Veterinaria y Zootecnia, Universidad Nacional Autónoma de México, Distrito Federal, México.

Carter G. 1984. Bacterial Identification. In: Diagnostic Procedures in Veterinary Bacteriology and Mycology. $4^{\text {th }}$ ed. Charles C Thomas Publisher, Springfield, USA, Pp 55-59.

Clavijo A, B Meléndez. 2002. Efecto del sistema de explotación sobre la aparición de mastitis caprina en dos fincas del estado Falcón, sus agentes etiológicos y la resistencia a antimicrobianos. Zoot Trop 20, 383-395.

Cremonesi P, B Castiglioni, G Malferrari, I Biunno, C Vimercati. 2006. Improved method for rapid extraction of mastitis pathogens directly from milk. J Dairy Sci 89, 168-169.

Figueroa ML. 2008. Cuenta de células somáticas en leche de cabra mediante las pruebas diagnósticas: Prueba de California, Wisconsin, cuenta microscópica y contador infrarrojo. Tesis licenciatura, Facultad de Medicina Veterinaria y Zootecnia, Universidad Nacional Autónoma de México,
Distrito Federal, México.

Gillespie B, S Oliver. 2005. Simultaneous detection of mastitis pathogens, Staphylococcus aureus, Streptococcus uberis and Streptococcus agalactiae by multiplex real-time polymerase chain reaction. J Dairy Sci 88, 3510-3518.

Hermans K, L Devriese, F Haesebrouk. 2004. Staphylococcus. In: Devrieses LA (ed). Pathogenesis of Bacterial Infections in Animals. $3^{\text {rd }}$ ed. Blackwell Publishing, Ames, USA, Pp 285-293.

Korman R. 1963. Coagulase-negative mutants of Staphylococcus aureus: genetic studies. J Bacteriol 86, 363-369.

Markoulatos P, N Siafakas, M Mocany. 2002. Multiplex polymerase chain reaction: a practical approach. J Clin Lab Anal 16, 47-51.

Mason W, J Blevins, K Beenken, N Wibowo, N Ojha, M Smelter. 2001. Multiplex PCR protocol for the diagnosis of staphylococal infection, J Clin Microbiol 39, 3332-3338.

Méndez A, R Pérez. 2004. La PCR múltiple en microbiología clínica. Enferm Infecc Microbiol Clin 22, 183-192.

Mosbath H, A Sayari, H Mejdoub, H Dhouib, Y Gargouri. 2005. Biochemical and molecular characterization of Staphylococcus xylosus lipase. BBA 1723, 282-291.

Phuektes P, D Mansell, F Browning. 2001. Multiplex polymerase chain reaction assay for simultaneous detection of Staphylococcus aureus and streptococcal causes of bovine mastitis. J Dairy Sci 84, 1140-1148.

Pyörälä S, S Taponen. 2009. Coagulase-negative staphylococciemerging mastitis pathogens. Vet Microbiol 134, 3-8.

Ruiz RRA. 2010. Desarrollo de una herramienta molecular (PCR multiplex) para la detección de los principales agentes de mastitis caprina. Tesis Maestría. Facultad de Medicina Veterinaria y Zootecnia, Universidad Nacional Autónoma de México, Distrito Federal, México.

Shearer J, B Harris. 2003. Mastitis in dairy goats. Institute of Food and Agricultural Sciences, University of Florida, Florida, USA.

Taponen S, Pyörälä S. 2009. Coagulase-negative staphylococci as cause of bovine mastitis - not so different from Staphylococcus aureus? Vet Micriobiol 134, 29-36.

Watanabe S, T Ito, T Sasaki, S Li, I Uchiyama, K Kishii. 2009. Genetic diversity of staphylocoagulase gene ( $\mathrm{coa})$ : insight into the evolution of variable chromosomal virulence factors in Staphylococcus aureus. PLoS ONE 4, e5714. 
